\title{
¿Regulación sectorial o normas de competencia?: El principio de supletoriedad en el caso Speedy Naked
}

Alessia Lercari Bueno ${ }^{(*)}$

Abogada por la Pontificia Universidad Católica del Perú.

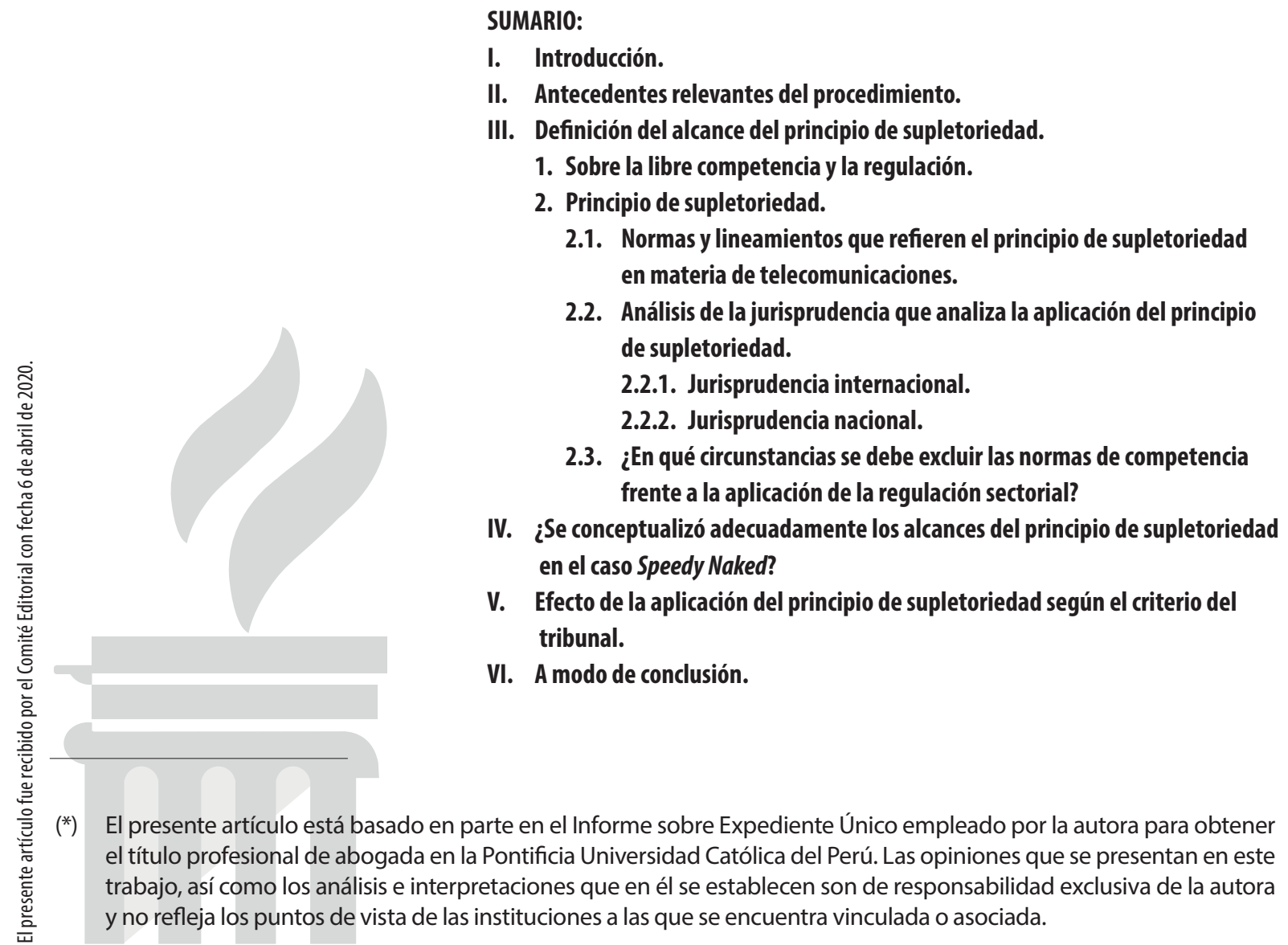




\section{RESUMEN:}

Se analizan los alcances del principio de supletoriedad en el Perú y se evalúa la definición de su ámbito de aplicación en el caso iniciado en contra de la empresa Telefónica del Perú S.A.A. por prácticas de abuso de posición de dominio en la modalidad de ventas atadas. Se propone una conceptualización del principio que busca resguardar la integridad del sistema jurídico en los casos de conflictos normativos entre regulación sectorial y normas de competencia.

Palabras clave: principio de supletoriedad, libre competencia, regulación, telecomunicaciones, conflicto normativo.

\footnotetext{
ABSTRACT:

This paper analyses the supplementary application of competition laws in Peru, and it assesses the criteria established in the procedure initiated against Telefónica del Perú S.A.A. for the alleged abuse of its dominant position in the form of tying. It outlines the scope for the supplementary application of competition laws and provides a definition that intends to preserve the integrity of the legal system when conflicts between regulation and competition arise.

Keywords: supplementary application of competition law, competition law, regulation, telecommunications, conflict of laws.
}

\section{INTRODUCCIÓN}

En el año 2011, el Organismo Supervisor de Inversión Privada en Telecomunicaciones -en adelante, "OSIPTEL" - inició un procedimiento sancionador en contra de Telefónica del Perú S.A.A. —en adelante, "Telefónica" — por la presunta comisión de una conducta de abuso de posición de dominio en la modalidad de ventas atadas. El procedimiento tenía por finalidad evaluar el esquema comercial utilizado por Telefónica para la comercialización del servicio de telefonía fija y del servicio de acceso a internet vía Asymmetric Digital Subscriber Line —en adelante, "ADSL" - . Hasta ese momento, la empresa venía comercializando ambos servicios de manera conjunta a través de un paquete denominado "Dúo: Speedy - Telefonía Fija".

De acuerdo con lo señalado por OSIPTEL, la empresa habría condicionado la venta del servicio de acceso a internet vía ADSL a la adquisición de una línea de telefonía fija, con la finalidad de aprovechar su posición privilegiada en el mercado de acceso a internet para resguardar su cuota en el mercado de telefonía fija, en el que venía perdiendo participación sostenidamente.

Este caso ha sido ampliamente estudiado para explorar temas tales como la aplicación del Derecho de la Competencia en el mercado de telecomunicaciones, y la figura de las ventas atadas como un abuso de posición de dominio en el Perú. Sin embargo, un aspecto menos estudiado es el conflicto que surge entre la aplicación de la regulación sectorial en materia de telecomunicaciones y las normas de competencia, así como la relevancia de definir adecuadamente el ámbito de aplicación del principio de supletoriedad como respuesta ante dicho conflicto.

Siendo ello así, el presente trabajo no se enfoca en determinar si la práctica comercial adoptada por Telefónica debió o no ser considerada como una infracción a las normas de competencia. Por el contrario, este trabajo se limita a analizar la definición del ámbito de aplicación del principio de supletoriedad por parte de OSIPTEL y su adecuación con la normativa y la jurisprudencia.

Como se desarrolla a mayor detalle en las siguientes líneas, es posible considerar que el criterio establecido por el Tribunal de Solución de Controversias de OSIPTEL —en adelante, el "Tribunal" - no fue consistente con la jurisprudencia ni con la experiencia comparada, generando mayores incongruencias con relación a la aplicación de este principio, así como mayor falta de predictibilidad para los administrados.

\section{ANTECEDENTES RELEVANTES DEL PRO- CEDIMIENTO}

El 27 de octubre de 2010, la Secretaría Técnica 
Adjunta de los Cuerpos Colegiados de OSIPTEL —en adelante, la "Secretaría Técnica" — inició una investigación de oficio con la finalidad de determinar si el esquema comercial utilizado por Telefónica para la venta de los servicios de acceso a internet vía ADSL y de telefonía fija constituiría una infracción al Decreto Legislativo 1034, Ley de Represión de Conductas Anticompetitivas —en adelante, la "Ley de Competencia"-.

Tras valorar el resultado de dicha investigación, mediante la Resolución N 001-2011-CCO/OSIPTEL del 13 de septiembre de 2011 —en adelante, la "Resolución Admisoria"-, el Cuerpo Colegiado resolvió disponer el inicio de un procedimiento sancionador en contra de Telefónica. La imputación se fundamentó en la presunta comisión de una infracción en la modalidad de venta atada con posibles efectos exclusorios en el mercado de telefonía fija, según lo establecido en el literal c) del artículo 10.2 de la Ley de Competencia'.

A lo largo del procedimiento, una de las principales defensas de Telefónica fue sostener que la conducta imputada no debía ser analizada bajo la óptica de las normas de competencia. Ello, en tanto el OSIPTEL había aprobado previamente el modelo comercial que era objeto de cuestionamiento. Siendo ello así, en tanto las normas de competencia solo debían aplicarse a las conductas que no se encontraran cubiertas por la regulación, en aplicación del principio de supletoriedad, correspondía desestimar la imputación formulada.

De acuerdo con lo señalado por Telefónica, la comercialización conjunta del servicio de acceso a internet vía ADSL al servicio de telefonía fija se encontraba autorizada a través de las aprobacio- nes a sus modelos de contratos de abonados del servicio Speedy —en adelante, los "Contratos de Abonados"- Dichos modelos contenían cláusulas expresas que requerían una línea de telefonía fija de Telefónica como condición adquirir el servicio Speedy. En ese sentido, la aceptación de dichos contratos constituyó una aprobación regulatoria que determinaría la exclusión de las normas de competencia a la comercialización conjunta de los servicios.

Tanto el Cuerpo Colegiado como el Tribunal desestimaron los argumentos de la empresa. Al respecto, el Tribunal señaló que, en los casos de aprobaciones regulatorias, el principio de supletoriedad aplicaría únicamente cuando existiera alguna norma que autorice expresamente una determinada práctica habiendo aplicado un proceso de revisión exhaustivo en términos de competencia.

Siendo ello así, se señaló que la aprobación a los Contratos de Abonados no podría implicar una aprobación al esquema comercial adoptado por Telefónica. El alcance del visto bueno emitido por el OSIPTEL en la aprobación de dichos contratos se limitó a constatar que éstos se adecuaban a la normativa sobre usuarios, y no efectuó un análisis de los efectos que podía generar la venta atada en la competencia.

Por tal motivo, el Cuerpo Colegiado y el Tribunal determinaron que sí correspondía aplicar las normas de competencia a la conducta que fue objeto de análisis, y tras realizar el análisis de competencia correspondiente, ambos órganos determinaron que la conducta debía ser considerada como una infracción a lo dispuesto en la Ley de Competencia.

Siendo ello así, mediante Resolución № 017-

1. Decreto Legislativo No. 1034, Ley de Represión de Conductas Anticompetitivas:

"Artículo 10.- El abuso de la posición de dominio.-

(...)

10.2. El abuso de la posición de dominio en el mercado podrá consistir en conductas de efecto exclusorio tales como:

(...)

c) Subordinar la celebración de contratos a la aceptación de prestaciones adicionales que, por su naturaleza o arreglo al uso comercial, no guarden relación con el objeto de tales contratos". 
2012-CCO/OSIPTEL del 20 de julio de 2012 -en adelante, la "Resolución del Cuerpo Colegiado"-, el Cuerpo Colegiado declaró fundada la denuncia de oficio emitida en contra de Telefónica, y dispuso sancionarla con una multa leve de 492.21 UIT —Unidades Impositivas Tributarias-. Asimismo, impuso como medida correctiva la obligación a ofrecer la venta del servicio de acceso internet vía ADSL solo —o naked-, evitando cualquier conducta que tenga efectos equivalentes a la atadura de los servicios.

Por su parte, el Tribunal confirmó lo resuelto por el Cuerpo Colegiado en cuanto a la existencia de una infracción y la medida correctiva impuesta mediante la Resolución N 004-2013TSC/OSIPTEL de fecha 31 de enero de 2013 en adelante, la "Resolución Final"—, pero optó por reducir la multa impuesta a un monto ascendente a 407 UIT. $^{2}$

Como se demuestra en la siguiente sección, el criterio empleado por el Cuerpo Colegiado y el Tribunal para resolver el presente caso no es consistente con la jurisprudencia ni con la experiencia comparada. A continuación, se propone una definición del alcance de dicho principio en función a las normas aplicables, la experiencia comprada y la jurisprudencia nacional.

\section{DEFINICIÓN DEL ALCANCE DEL PRINCI- PIO DE SUPLETORIEDAD}

\section{Sobre la libre competencia y la regulación.}

De acuerdo con la teoría económica, la com- petencia es el mecanismo más adecuado para promover la asignación eficiente de recursos en el mercado. A través del libre juego de la oferta y la demanda, los consumidores pueden obtener beneficios tales como la disminución de precios, la mejora en la calidad de los productos y el aumento de opciones de consumo. ${ }^{3}$

Por ello, la defensa de la libre competencia es una de las políticas públicas más importantes para el desarrollo económico de un país. Tal es así que la Constitución Política del Perú dispone un régimen especial de protección al señalar que: "(e)I Estado facilita y vigila la libre competencia. Combate toda práctica que la limite y el abuso de posiciones dominantes o monopólicas". ${ }^{4} \mathrm{EI}$ desarrollo legislativo de dicho precepto se encuentra en la Ley de Competencia, la cual establece en su artículo 1 que la protección otorgada tiene por finalidad "promover la eficiencia económica en los mercados para el bienestar de los consumidores". ${ }^{5}$

Así, el Derecho de la Competencia protege y potencia el proceso competitivo frente a conductas que puedan restringirlo u obstaculizarlo. ${ }^{6}$ En la mayoría de los casos, la normativa de competencia cumple con dicha finalidad a través de mecanismos de intervención ex post ${ }^{7}$, es decir, mediante el cuestionamiento y la prohibición de conductas que ya han tomado lugar en el mercado, a través de procedimientos administrativos sancionadores.

Es importante notar que estas normas solo pueden generar eficiencias en los mercados

2. Telefónica interpuso una demanda contencioso-administrativa en contra de la Resolución Final. A la fecha de la elaboración del presente trabajo, dicho proceso aún se encuentra pendiente de resolución. Asimismo, de manera posterior a la emisión de la Resolución Final, se inició un procedimiento por incumplimiento a la medida correctiva impuesta en el presente caso. Mediante Resolución № 011-2016-TSC/OSIPTEL de fecha 16 de diciembre de 2016, se determinó que la empresa había incumplido lo ordenado mediante la Resolución Final y se optó por imponer una sanción de 300 UIT. Dicho pronunciamiento también ha sido cuestionado vía proceso contencioso-administrativo y se mantiene pendiente de resolución.

3. Richard Whish y David Bailey, Competition Law (Oxford: Oxford University Press, 2015), 4.

4. Artículo 61 de la Constitución Política del Perú.

5. Artículo 1 de la Ley de Competencia. 
que cuenten con condiciones mínimas que permitan el desarrollo de la competencia. En efecto, existen diversos mercados que presentan fallas derivadas de la imposibilidad de que se genere, o que se aumente sustancialmente la competencia. Esto puede ocurrir por motivos tales como la existencia de monopolios naturales o de elevados índices de concentración. Tales desperfectos impiden que los mercados funcionen eficientemente sin intervención previa, y por ello, dichas fallas deben ser subsanadas a través de regulación.

Por ejemplo, en el mercado de las telecomunicaciones los operadores suelen contar con posiciones privilegiadas al ser titulares de la infraestructura esencial para la prestación de los servicios - por ejemplo, Telefónica tiene control del bucle local, lo que la coloca en una mejor posición para la prestación del servicio de internet vía ADSL- La existencia de estas situaciones genera la necesidad de establecer mandatos o lineamientos de conducta a los agentes económicos a fin de generar artificialmente la eficiencia que se obtendría en situación de competencia. ${ }^{8}$

Siendo ello así, la regulación puede definirse como:

“(...) una acción deliberada del Estado utilizando mecanismos de Derecho Público, dirigida a influir sobre las actividades económicas realizadas en los mercados para conseguir objetivos de interés público, normando, supervisando, vigilando, sancionando $y$ eventualmente, resolviendo controversias entre los sujetos regulados." ${ }^{\prime 9}$

En términos generales, la regulación refiere a los actos voluntarios del Estado que tienen por finalidad influir en el mercado de manera previa a la actuación de los agentes. Es decir, se trata de una herramienta ex ante de intervención, con la cual las normas se emiten para guiar la conducta de los agentes económicos en el mercado.

Tradicionalmente, la regulación toma dos formas de acuerdo con los bienes jurídicos protegidos por la misma. Por un lado, se tiene la regulación económica, referida a la intervención sustitutiva del Estado que tiene por finalidad simular la existencia de condiciones competitivas en los mercados que presentan las fallas previamente referidas derivadas de la ausencia o escases de competencia. ${ }^{10}$

Pero, por otro lado, se tiene la regulación social, definida como la intervención del Estado que tiene por objetivo "lograr un equilibrio entre los derechos de los ciudadanos, evitando que las consecuencias del ejercicio de determinados derechos privados perjudiquen a los derechos de terceros los cuales se formalizan como intereses públicos o de

6. Eduardo Quintana, Análisis de las funciones del Indecopi a la luz de las decisiones de sus órganos resolutivos - Libre Competencia (Lima: Instituto Nacional de Defensa de la Competencia y de la Protección de la Propiedad Intelectual (Indecopi), 2013), 9.

7. Si bien existe control ex ante constituido por el control de concentraciones, a la fecha, dicho control se encuentra limitado únicamente al sector eléctrico.

8. Joselyn Olaechea, «Libre Competencia versus Regulación: Sobre la Aplicación del Principio de Supletoriedad en la Nueva Ley de Represión de las Conductas Anticompetitivas - LRCA», Revista de la Competencia y la Propiedad Intelectual, n. 13 (2011): 62.

9. Robert Baldwin, Martin Cave y Martin Lodge, Understanding Regulation: Theory, Strategy, and Practice (Oxford: Oxford University Press, 2012), 2.

10. Ramón Huapaya, «Algunos apuntes sobre las relaciones entre el Derecho Administrativo Económico y el concepto anglosajón de la Regulación», lus Et Veritas, n. 40 (2010): 312. 
la colectividad..$^{m 1}$ Este tipo de regulación tiene por finalidad corregir las fallas que se derivan en situaciones donde el ejercicio de derechos individuales puede afectar el bienestar de la colectividad. Así, la regulación social refiere a materias tales como seguridad, salud, medio ambiente, protección al consumidor, entre otras.

Con el propósito de dinamizar los mercados y fomentar la competencia, en los últimos años, las industrias altamente reguladas han sido objeto de procesos de "desregulación". Este concepto se refiere a:

"la política por la que los Estados eliminan o reducen considerablemente su intervención en el desarrollo de las actividades productivas, suprimiendo parte de las disposiciones legales existentes con el objeto de que se incremente el tráfico económico originado en la iniciativa privada."12

No obstante, algunos mercados no pueden presentar, de manera espontánea, condiciones de competencia, y es a partir de este hecho que la regulación constituye un mecanismo para fomentar dichas condiciones en los mercados. En este contexto, surge una nueva modalidad de regulación denominada regulatory antitrust o regulación para la competencia. Estas normas parten de la premisa — o paradoja - de que "la regulación se ha convertido en el principal instrumento que el Estado emplea para introducir competencia en las industrias monopólicas. ${ }^{13 "}$

Si bien existen espacios en los que la regulación y las políticas de competencia se complementan - como en el regulatory antitrust-, existen también espacios en los que éstas se sobrepo- nen. En efecto, es posible que las normas de competencia busquen controlar conductas que ya han sido aprobadas o prohibidas por la regulación. Es en estos casos en los que la aplicación de los principios generales del Derecho proporciona un mecanismo para aliviar dichos conflictos a través del principio de supletoriedad.

\section{Principio de supletoriedad.}

El principio de supletoriedad brinda una solución a las situaciones en las que existe un conflicto en la aplicación de la regulación sectorial frente a la aplicación de normas de libre competencia. Dicha solución implica la adopción del criterio de especialidad de las normas como principio general del Derecho bajo el cual "la norma especial prima sobre la norma general".

En dicho contexto, la regulación se aplica de manera prioritaria al tener carácter especial, mientras que, por otro lado, las normas de competencia aplican de manera residual en atención a su carácter general. En ese sentido, las normas de competencia "aplicarán siempre que no exista una regulación específica que haya tratado el problema concreto de manera directa." ${ }^{\prime 4}$

Sin perjuicio de que el principio de supletoriedad se constituye como una expresión de los principios generales del Derecho, las normas aplicables al sector telecomunicaciones -así como otras industrias reguladas-, así como la Ley de Competencia, introducen este concepto en la regulación y las normas de competencia.

\subsection{Normas y lineamientos que refieren el principio de supletoriedad en materia de telecomunicaciones. ${ }^{15}$}

11. Ibíd.

12. Eduardo Quintana, «¿Es la política de la competencia "supletoria" de la regulación de Telecomunicaciones?», Ius Et Veritas, n. 27 (2003): 99.

13. Ramón Huapaya, Op. Cit., 40.

14. Joselyn Olaechea, Op. Cit., 77.

15. Es importante notar que en el presente trabajo solo se abordará la normativa en el sector telecomunicaciones en la medida que es el mercado en el que se produce el caso que motiva este estudio. 
La primera vez que se introdujo el principio de supletoriedad en el sector telecomunicaciones fue en el año 1994, con la emisión del Reglamento General de la Ley de Telecomunicaciones, aprobado por Decreto Supremo No 06-94-TCC —en adelante, el "Reglamento de la Ley de Telecomunicaciones"-. Mediante dicha norma, se incorporó el principio al establecerse lo siguiente:

"Artículo 229.- En lo que no esté previsto en la Ley y el Reglamento, en lo referido a la prohibición de las prácticas empresariales restrictivas de la libre competencia, se aplicarán las disposiciones legales vigentes sobre prácticas monopólicas controlistas y restrictivas de la libre competencia."

Posteriormente, en el año 1998, los Lineamientos de Política de Apertura del Mercado de Telecomunicaciones del Perú aprobados por Decreto Supremo No 020-98-MTC —en adelante, los "Lineamientos de Apertura de Mercado" - precisaron lo dispuesto en el Reglamento de la Ley de Telecomunicaciones, estableciendo lo siguiente:

"111. Para la promoción y preservación de la libre y leal competencia en el mercado de los servicios públicos de telecomunicaciones, los órganos competentes del Sector aplicarán la normativa sectorial específica $y$, supletoriamente, en cuanto sean pertinentes, las disposiciones de los Decretos Legislativos Nos. 701 y 807, las del Decreto Ley No 26122 y disposiciones modificatorias, complementarias, ampliatorias y conexas."

En el año 2000, los Lineamientos Generales para la Aplicación de las Normas de Libre Competencia en el Ámbito de las Telecomunicaciones, aprobados mediante Resolución № 003-2000CD/OSIPTEL —en adelante, los "Lineamientos de Libre Competencia" - precisaron con mayor exactitud los alcances del principio. Es importante notar que, si bien estos lineamientos no resultan de aplicación obligatoria, su relevancia recae en el hecho de que recogen los criterios de interpretación utilizados por los órganos resolutivos en la solución de casos. En ese sentido, se estableció lo siguiente:
"2.1 (...) Las normas generales de libre competencia están concebidas, en principio, en el marco de mercados no regulados de manera específica.

(...)

4. (...) OSIPTEL entiende que para la promoción y preservación de la libre competencia en el mercado de las telecomunicaciones, se aplicará la normativa específica del sector y de manera supletoria la normativa general de libre competencia.

(...)

Esto implica que OSIPTEL aplicará las normas generales de libre competencia en el supuesto que una determinada práctica o conducta no esté tipificada o cubierta de manera expresa por la normatividad específica del sector, pero genere, sin embargo, efectos anticompetitivos. (...)

Esto no impide que OSIPTEL aplique las normas de libre competencia en aquellos casos que no estén expresamente prohibidos o en aquellos casos en los que los principios de interpretación permitan una más adecuada aplicación de la referida normativa."

Como se puede apreciar, los Lineamientos de Libre Competencia añadieron una condición adicional a las contenidas en el Reglamento de la Ley de Telecomunicaciones y los Lineamientos para la Apertura del Mercado. De acuerdo con lo establecido en los Lineamientos de Libre Competencia, para que proceda la exclusión de las normas de competencia, la conducta objeto de análisis deberá encontrarse cubierta o tipificada de manera expresa por la regulación.

Por otro lado, en el año 2001, el Reglamento General de OSIPTEL, aprobado por el Decreto Supremo No 008-2001-PCM —en adelante, el "Reglamento General de OSIPTEL" — ratificó los criterios previamente mencionados señalando lo siguiente:

"Artículo 12. Principio de supletoriedad. Las normas de libre competencia son supletorias a las disposiciones normativas y/o regulatorias que dicte el OSIPTEL en el ámbito de su competencia. En caso de conflicto primarán las disposiciones dictadas por el OSIPTEL."

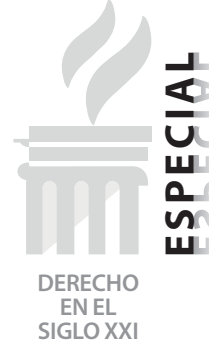


Finalmente ${ }^{16}$, fue emitida la Ley de Competencia, la cual, a diferencia del Decreto Legislativo 701 que elimina las prácticas monopólicas, controlistas y restrictivas de la competencia —en adelante, la "Ley Antimonopolio"—, sí estableció referencia expresa al principio, señalando:

\begin{abstract}
"Artículo 30.- Ámbito de aplicación objetivo.Se encuentra fuera de aplicación de la presente Ley aquella conducta que es consecuencia de lo dispuesto en una norma legal. El cuestionamiento a dicha norma se realizará mediante las vías correspondientes y no ante la autoridad de competencia prevista en la presente Ley. El Estado podrá asumir las acciones que considere necesarias para contribuir a mejorar las condiciones de oferta de los productos en beneficio de los consumidores."
\end{abstract}

En consecuencia, se puede observar que la normativa que se encontraba vigente en el momento en el que fue emitida la Resolución Final es concordante en señalar que siempre que una norma regule una conducta de manera expresa, sea admitiéndola o prohibiéndola, se excluirá la aplicación de las normas de competencia. No obstante, si bien las normas son concordantes en la regla aplicable, éstas no otorgan una respuesta específica para el diverso tipo de conflictos que pueden presentarse. Es por tal motivo que, para terminar de delimitar los alcances del principio, corresponde analizar la casuística en la materia.

\subsection{Análisis de la jurisprudencia que analiza la aplicación del principio de supletoriedad.}

\subsubsection{Jurisprudencia internacional.}

Los conflictos que surgen entre la aplicación de la regulación económica y las normas de competencia se han presentado tanto en la Unión Europea como en Estados Unidos. Siendo ello así, en la presente sección se analiza brevemente las soluciones que se han brindado en dichas jurisdicciones.

El pronunciamiento europeo que estableció el criterio aplicable frente a dicho conflicto se encuentra en el caso Deutsche Telekom. ${ }^{7}$ En dicha oportunidad, la Comisión de las Comunidades Europeas —en adelante, la "Comisión Europea" - inició un procedimiento en contra de la empresa alemana Deutsche Telekom por la presunta violación al artículo 82 del Tratado de la Comunidad Europea al incurrir en una conducta anticompetitiva en la modalidad de estrechamiento de márgenes. ${ }^{18}$

No obstante, los precios fijados por Deutsche Telekom que fueron objeto de cuestionamiento habían sido aprobados previamente por el regulador alemán, el cual había establecido tarifas topes. De acuerdo con la decisión de la Comisión Europea, en la medida que la empresa mantenía autonomía sobre la decisión final en la fijación de precios - esto es, podía fijar libremente dentro del margen autorizado por la entidad regulatoria-, su comportamiento podía tener como consecuencia la infracción a las normas de competencia. En ese sentido, se sostuvo lo siguiente:

"(...) la aplicación de la normativa de competencia no está excluida, según jurisprudencia

16. Se debe notar que, de manera posterior a la resolución del caso que es objeto de análisis mediante el presente trabajo, el Consejo Directivo del OSIPTEL emitió los Lineamientos Generales para la Aplicación de las Normas de Represión de Conductas Anticompetitivas y Desleales en el Ámbito de las Telecomunicaciones, aprobados por Resolución Nº77-2016-CD/OSIPTEL. En dichos lineamientos, se adoptó la interpretación establecida en la Resolución Final, la cual será analizada a mayor detalle en los siguientes acápites.

17. Comisión de las Comunidades Europeas, Asunto COMP/C-1/37.451, 37.578, 37.579 - Deutsche Telekom AG. Esta decisión fue confirmada por el Tribunal de Justicia de la Unión Europea mediante sentencia del 14 de octubre de 2010, Asunto C-280/08.

18. Dicha conducta implicó el cobro de un precio más elevado por el acceso a infraestructura en el mercado aguas arribas frente a los precios en el mercado aguas abajo. 
reiterada del Tribunal de Justicia de las Comunidades Europeas y del Tribunal de Primera Instancia de las Comunidades Europeas, si las disposiciones sectoriales pertinentes no impiden que las empresas sujetas a tales disposiciones adopten un comportamiento autónomo que pueda obstaculizar, restringir o falsear la competencia." ${ }^{19}$

En ese sentido, mediante la emisión de la decisión citada, la Comisión Europea ha optado por priorizar la aplicación de las normas de competencia comunitarias sobre las normas regulatorias nacionales. ${ }^{20}$ Ello, siempre que las empresas puedan mantener cierto nivel de autonomía en la realización de las conductas que pueden ser anticompetitivas.

De acuerdo con autores como Monti, la posición de la Comisión Europea se sustenta en el hecho de que la aplicación de las normas de competencia sobre la regulación nacional permite "corregir las debilidades de los reguladores nacionales." ${ }^{21}$ En efecto, en el caso Deutsche Telekom, la Comisión Europea opta por "corregir" un acto que generaba daños a la competencia, y que había sido habilitado por el regulador nacional.
Sin perjuicio de ello, la Corte Suprema de los Estados Unidos de América -en adelante, la Corte Suprema estadounidense- ha establecido un criterio distinto al mencionado. En el caso Trinko ${ }^{22}$, una demanda fue presentada por un cliente de la operadora AT\&T en Nueva York -en representación de la clase, un conjunto de demandantes - en contra de Verizon, la empresa dominante local en la prestación del servicio de telefonía fija. El demandante alegó que Verizon se había negado injustificadamente a permitir a AT\&T el acceso a sus sistemas, lo cual generaba un perjuicio a la competencia, y particularmente, a los clientes de AT\&T.

De acuerdo con la demanda planteada, dicha negativa generaba una infracción tanto a la Sherman Act, ley de competencia estadounidense, como a la Telecommunications Act, ley de telecomunicaciones de dicho país. En el pronunciamiento final emitido por la Corte Suprema estadounidense en enero de 2004, se dispuso que no era posible considerar que la conducta consistía una infracción a la Sherman Act, en tanto ésta se encontraba regulada en la Telecommunications Act. ${ }^{23}$

Asimismo, de manera posterior, mediante la re-

19. Ibíd.

20. Pierre Larouche, «Contrasting legal solutions and the comparability of EU and US experiences», TILEC Discussion Papers, n. ${ }^{\circ}$ 2006-028 (2006): 7.

21. Giorgio Monti, «Managing the Intersection of Utilities Regulation and EC Competition Law», Society and Economy Working Papers 8/2008, (2008): 11.

Traducción libre de: "Therefore, from a policy perspective, the need for institutional reform serves as a justification for the status quo: competition law applies even when it contradicts national regulatory policy in order to correct the weakness of national regulators today".

22. Corte Suprema de los Estados Unidos de América, Verizon Communications Inc. contra Law Offices of Curtis V. Trinko, 2004.

23. Ibíd. "Un factor de particular importancia es la existencia de una estructura regulatoria diseñada para desincentivar y remediar el daño anticompetitivo. Cuando una estructura de este tipo existe, el beneficio adicional que ofrece la aplicación de las normas de competencia tenderá a ser pequeño, y será menos verosímil que las normas de competencia contemplen ese escrutinio adicional. Por el contrario, cuando no existan elementos en la estructura regulatoria que cumplan con la función de defensa de la competencia, el beneficio de la aplicación de las normas de competencia será mayor a las desventajas considerables que en ocasiones original". 
solución del caso Credit Suisse ${ }^{24}$, la Corte Suprema estadounidense estableció los siguientes criterios que deben ser tomados en consideración para determinar la exclusión de las normas de competencia:

"(1) la existencia de una autoridad reguladora facultada legalmente para supervisar las actividades cuestionadas; (2) evidencia de que la autoridad reguladora ejerce dicha facultad; (3) un riesgo consistente en que la aplicación conjunta de la regulación económica y las normas de competencia generará directivas, requerimientos, obligaciones, derechos y/o estándares de conducta opuestos; $y$, (4) el conflicto generado afecta prácticas que constituyen aspectos centrales del objeto de la regulación." ${ }^{25}$

El criterio establecido en los casos citados se denomina la doctrina de la inmunidad implícita y señala que cuando la regulación refiera a una actividad que podría cuestionarse mediante la aplicación de las normas de competencia, estas últimas deberán ser excluidas. ${ }^{26}$ Siendo ello así, se observa que Estados Unidos adopta un enfoque más amplio de exclusión. Es razonable entender la diferencia de enfoques entre las jurisdicciones mencionadas, si se tiene en consideración que, en el caso de Estados Unidos, no existe la necesidad de "corregir" errores regulatorios nacionales, como sí ocurre en la Unión Europea.
Tomando en consideración las opciones adoptadas por la experiencia internacional, corresponde evaluar los pronunciamientos adoptados por las autoridades peruanas.

\subsubsection{Jurisprudencia nacional.}

De manera previa a la emisión del pronunciamiento que es objeto de análisis, en el Perú ya se han presentado casos en los que se ha denunciado mediante procedimientos de libre competencia conductas que se encontraban cubiertas por la regulación sectorial; esto es, que se encontraban prohibidas o autorizadas previamente por medio de regulación. En un inicio, la aplicación del principio de supletoriedad como solución para dichas situaciones fue bastante uniforme; sin embargo, como se señala en la presente sección, los criterios más recientes son los que introdujeron discordancias en la aplicación del criterio.

En el año 2002, se resolvieron tres procedimientos de libre competencia en los que se optó por no aplicar la Ley Antimonopolio en la medida que las conductas objeto de imputación se encontraban comprendidas por la regulación. En dichas oportunidades, se buscó cuestionar incumplimientos a normas regulatorias mediante procedimientos de libre competencia. Por tal motivo, las denuncias fueron declaradas improcedentes.

Traducción libre de: "One factor of particular importance is the existence of a regulatory structure designed to deter and remedy anticompetitive harm. Where such a structure exists, the additional benefit to competition provided by antitrust enforcement will tend to be small, and it will be less plausible that the antitrust laws contemplate such additional scrutiny. Where, by contrast, 't $t$ ] here is nothing built into the regulatory scheme which performs the antitrust function,' the benefits of antitrust are worth its sometimes considerable disadvantages".

24. Corte Suprema de los Estados Unidos de América, Credit Suisse Securities LCC contra Billing, 2007.

25. Ibíd.

Traducción libre de: "(1) the existence of regulatory authority under [the relevant statute] to supervise the activities in question; (2) evidence that the responsible regulatory entities exercise that authority; (...) (3) a resulting risk that the [relevant law] and antitrust laws, if both applicable, would produce conflicting guidance, requirements, duties, privileges, or standards of conduct [; and] (...) (4) (...) the possible conflict affected practices that lie squarely within an area (...) that the [relevant statute] seeks to regulate."

26. Barak Orbach, «The Implied Antitrust Immunity», Arizona Legal Studies Discussion Paper, n. ${ }^{\circ} 14-16$ (2014): 3. 
En el caso resuelto mediante la Resolución $\mathrm{N}^{\circ}$ 006-2002/CLC-INDECOPI del 13 de marzo de 2002, Napsa denunció a Edelnor por la presunta negativa injustificada del suministro eléctrico de 0,30 Kw para el funcionamiento de dos paneles publicitarios ubicados dentro de la zona de concesión de Edelnor. Sin embargo, la Comisión de Defensa de la Libre Competencia del Instituto Nacional de Defensa de la Competencia y de la Protección de la Propiedad Intelectual —en adelante, la "Comisión de Libre Competencia" - declaró improcedente la denuncia pues la Ley de Concesiones Eléctricas, Decreto Ley 25844 , disponía la obligación de las distribuidoras eléctricas de proveer energía a todos los usuarios que empleen menos de $1 \mathrm{Mw}$ de potencia dentro de su zona de concesión. En ese sentido, al existir una obligación general de abastecimiento, correspondía al regulador OSINERGMIN - Organismo Supervisor de la Inversión en Energía y Minería-, al momento en el que se produjo la controversia, conocer la denuncia por infracción a la normativa sectorial.

De manera similar, en el caso resuelto mediante la Resolución N 052-2002-CCO/OSIPTEL del 16 de julio de 2002, AT\&T denunció a Telefónica por un abuso de posición de dominio en la modalidad de negativa injustificada. La denuncia se sustentó en que Telefónica se había negado a programar la numeración "133", correspondiente a la denunciante, en su red. Dicha programación resultaba necesaria para AT\&T pudiera ofrecer el servicio de llamadas telefónicas locales y de larga distancia mediante el uso de tarjetas prepago. No obstante, tal y como sucedió en el caso referido previamente, el Cuerpo Colegiado desestimó la denuncia en tanto la regulación del Ministerio de Transportes y Comunicaciones y de OSIPTEL establecían un mecanismo para el acceso de operadores a las redes del concesionario local. En ese sentido, la negativa de Telefónica no podía ser analizada bajo las normas de libre competencia, puesto que existía un procedimiento de índole regulatorio que debía aplicarse en el caso.

Finalmente, en ese mismo año también fue declarado improcedente el procedimiento resuelto mediante la Resolución $N^{\circ}$ 015-2002/
CLC-INDECOPI del 28 de agosto de 2002 iniciado por Practimar. Practimar era una empresa dedicada a la administración de personal que prestan servicios en el Terminal Portuario de llo. La empresa había contratado con Opemarpo la prestación de tales servicios. Sin embargo, la empresa Empresa Nacional de Puertos S.A. en adelante, "ENAPU" - se negó a permitir la entrada del personal de Opemarpo al Terminal Portuario de llo. Por lo tanto, Practimar interpuso una denuncia en su contra, calificando su conducta como una negativa injustificada de trato. Sin embargo, la regulación sectorial en materia portuaria establecía un procedimiento para el otorgamiento de acceso a la infraestructura esencial de ENAPU. En ese sentido, correspondía que el Organismo Supervisor de la Inversión en Infraestructura de Transporte de Uso Público -OSITRAN - conozca cualquier incumplimiento relativo a dicho procedimiento, y el Instituto Nacional de Defensa de la Competencia y de la Protección de la Propiedad Intelectual —INDECOPI — no calificaba como entidad competente para conocer la denuncia.

Como se puede observar, los casos referidos presentaron características ampliamente similares, y por tal motivo, los pronunciamientos que desestimaron las imputaciones formuladas emplearon criterios de solución homogéneos. Siempre que se negara la realización de una conducta que estaba dispuesta en la normativa sectorial como obligación, no resultaba procedente la interposición de una denuncia en la modalidad de abuso de posición de dominio.

Pocos años después, se presentaron dos nuevos casos en materia de telecomunicaciones en los que los hechos ameritaron el análisis de un nuevo tipo de conflicto entre la regulación y las normas de competencia. En tales situaciones se debió evaluar la aplicación de las normas de competencia a conductas que ya se habrían encontrado autorizadas por los reguladores.

Mediante la Resolución $\mathrm{N}^{\circ}$ 002-2004-CCO/ OSIPTEL del 13 de agosto de 2004, Red Privada Virtual denunció a Telefónica por la presunta adopción de una práctica discriminatoria mediante la comercialización del plan tarifario 
denominado "Línea Premium". De acuerdo con la denunciante, la discriminación se habría producido en tanto los proveedores de internet no conectados a la red de telefonía fija local de Telefónica no podían acceder a la tarifa plana promocional, a diferencia de los proveedores que sí se encontraban conectados a través de su red.

Como sucedió en los casos previos, el Cuerpo Colegiado desestimó la denuncia formulada. En efecto, la regulación disponía que correspondía a la Gerencia General de OSIPTEL aprobar los planes promocionales tarifarios de las empresas operadoras, lo que determinada que el plan objeto de imputación ya había recibido aprobación regulatoria en dicha oportunidad. En ese sentido, no correspondía analizar el potencial efecto anticompetitivo de la conducta en tanto la empresa había actuado bajo el amparo de la aprobación emitida por la Gerencia General.

Asimismo, mediante las Resoluciones $\mathrm{N}^{\circ}$ 0082005-CCO/OSIPTEL del 11 de marzo de 2005 y $\mathrm{N}^{\circ}$ 0006-2005-TSC/OSIPTEL del 21 de abril de 2005, tanto el Cuerpo Colegiado como el Tribunal debieron pronunciarse respecto de una denuncia interpuesto por Nextel en contra de Telefónica Móviles por la realización de una práctica con características similares. La denuncia se sustentó en el hecho de que el costo de los cargos de terminación en la red de la denunciada era significativamente mayor para sus competidores, frente a la tarifa que dicha empresa cobraba para las comunicaciones dentro de su red -on-net-. Los órganos resolutivos de OSIPTEL determinaron que no podían pronunciarse respecto a los cargos de terminación, ya que estos habían sido pactados entre las partes mediante un procedimiento que había culminado con suscripción de un contrato de interconexión aprobado por la Gerencia General de OSIPTEL.

Como se observa, al presentarse un nuevo tipo de conflictos, esto es, la aplicación de normas de competencia a conductas que ya se encontraban autorizadas por la regulación sectorial, la autoridad optó por brindar primacía a las normas regulatorias. Todo ello, en concordan- cia con los criterios establecidos en las normas que regularon el principio de supletoriedad.

No obstante, si bien la aplicación del principio de supletoriedad se venía presentando de manera uniforme, la Sala de Defensa de la Competencia de INDECOPI —en adelante, la "Sala"se apartó de los criterios señalados.

En el caso resuelto mediante Resoluciones $\mathrm{N}^{\circ}$ 021-2007/CLC-INDEOCPI del 21 de marzo de 2007 y Nº 0004-2008/TDC-INDECOPI del 3 de enero de 2008, Alfagas denunció a Repsol y otras empresas competidoras por una presunta negativa injustificada de trato, en tanto éstas se negaban a efectuar canjes de balones de gas, a pesar de que la denunciante lo había requerido en múltiples ocasiones.

Las condiciones de intercambio y comercialización de cilindros se encontraban dispuestas en la regulación sectorial de OSINERGMIN. Por tal motivo, de acuerdo con lo resuelto en primera instancia correspondía que dicho regulador conozca la conducta y, por tal motivo, la Comisión de Libre Competencia declaró improcedente la denuncia. Sin embargo, en segunda instancia, la Sala anuló la decisión bajo el argumento que solo la regulación económica, y no la social, podía desplazar la aplicación de normas de competencia. En ese sentido, en la medida que el mercado de hidrocarburos no es monopólico, las normas aplicables a dicho sector no podían ser consideradas como regulación económica. Siendo ello así, al tratarse de un conflicto que involucraba regulación social, ésta podía ser aplicada de manera simultánea con las normas de competencia.

Como se puede apreciar, la jurisprudencia fue consistente en brindarle primacía a la regulación sectorial de manera sistemática, hasta la resolución del caso Alfagas. La introducción de dicho criterio determinó un quiebre en la interpretación que venía siendo aplicada al principio de supletoriedad. Sin perjuicio de ello, después de haber analizado las normas aplicables, así como la jurisprudencia, en la siguiente sección se propone una delimitación al ámbito de aplicación del principio. 


\section{3. ¿En qué circunstancias se debe excluir las normas de competencia frente a la apli- cación de la regulación sectorial?}

Para determinar un criterio o solución uniforme, es necesario determinar los tipos de conflictos que pueden presentarse en la aplicación de regulación sectorial y normas de competencia. Al analizar tales tipos de conflictos, será posible distinguir entre las distintas soluciones disponibles.

Después de observar la jurisprudencia en la materia, es posible definir que existen dos tipos de conflictos que pueden presentarse en el análisis de conductas en el mercado: (a) conductas que se encuentran aprobadas o permitidas por las normas sectoriales, y que son tipificadas por las normas de competencia y (b) conductas que se encuentran tipificadas por normas sectoriales, así como por las normas de competencia.

Para el caso de la situación descrita en el numeral (a), tanto la doctrina como la jurisprudencia es pacífica en señalar que, en tal escenario, siempre se debe excluir la aplicación de las normas de competencia. Ello, debido a que:

"como parte de las funciones que la Ley le ha encomendado al regulador, la autorización de conductas que afecten a la competencia implica dar prioridad a otros objetivos que resulten de sumo valor para el desarrollo de una industria determinada en un plazo definido."27

Aceptar una respuesta distinta generaría situaciones en la que los agentes se encontrarían frente a una encrucijada en la que deben decidir si corresponde vulnerar la regulación sectorial o de vulnerar las normas de competencia ${ }^{28}$, así como en situaciones de impredecibilidad sobre la validez de las autorizaciones regulatorias.

Se debe tener en consideración que, para que concurra la exclusión de las normas de competencia, la conducta que es permitida o aprobada por la regulación sectorial deberá señalarse de manera expresa, según lo establecido en los Lineamientos de Libre Competencia citados previamente. ${ }^{29}$ Del mismo modo, a fin de evitar colocar a los agentes económicos en situaciones de conflicto nuevamente, deberá será posible que la conducta que excluye la aplicación de las normas de competencia también podrá ser aquella que es esencialmente necesaria para ejecutar una conducta expresamente ordenada.

Por otro lado, en el caso de las situaciones descritas en el numeral (b), se concluye que, independientemente de la naturaleza de la regulación aplicable al caso, deberá optarse también por excluir la aplicación de las normas de competencia. No obstante, para implementar esta propuesta deberá dejarse sin efecto el criterio establecido en el caso Alfagas.

En el caso Alfagas, la Sala estableció que, ante conflictos normativos, solo deberán excluirse las normas de competencia cuando éstas entren en conflicto con "regulación económica". En ese sentido, de acuerdo con dicho pronunciamiento, existe la posibilidad de aplicar concurrentemente las normas de competencia junto con la "regulación social"30 puesto que

27. Joselyn Olaechea, Op. Cit., 90.

28. Carlos Patrón, «El que mucho abarca, poco aprieta: del llamado principio de supletoriedad en materia de libre competencia», Derecho \& Sociedad, n. 30 (2008): 406

29. Numeral 4 de los Lineamientos de Libre Competencia.

30. Es importante notar que uno de los aspectos más cuestionados del caso Alfagas es la definición incorporada por la Sala de los conceptos de regulación económica y regulación social. De acuerdo con lo señalado por la Sala, la regulación social se define como aquella aplicable a los mercados que "no son monopólicos". Consecuentemente, se determinó que las disposiciones relativas al intercambio de cilindros deberán ser consideradas como regulación social en vista de que aplicaría en un mercado en el que existe competencia. 
"(e)I sistema jurídico admite que un mismo hecho pueda tener distintas consecuencias jurídicas, de forma tal que a partir de una conducta específica se puedan derivar diversas infracciones y sanciones igualmente diversas." ${ }^{131}$

No obstante, en los casos en los que existe regulación sectorial prohibiendo una conducta, no será pertinente aplicar las normas de competencia, en tanto éstas últimas tienen carácter general y no han sido emitidas para un sector particular. Aún cuando se trate de un conflicto entre normas de competencia y regulación que podría ser considerada de carácter social, la aplicación del principio general del Derecho sobre la especialidad normativa determinaría optar por la exclusión del régimen general. Lo contrario implicaría aceptar la posibilidad de que una conducta sea sancionada dos veces por la vía administrativa.

En efecto, el hecho de que una conducta se encuentre prohibida por la regulación sectorial determina que ésta no deba producirse en el mercado. No obstante, de ser el caso, la vía para cuestionar y sancionar su concurrencia debe ser, únicamente, la que se encuentra dispuesta en la norma especial que la prohíbe. Con ello, se elimina la posibilidad de que se produzca un escenario de doble prohibición.

Es importante notar que las normas y lineamientos que regulaban la aplicación del principio de supletoriedad no establecieron precisiones ni diferenciaciones en cuanto a la naturaleza de la regulación que excluye la aplicación de normas de competencia. Particularmente, en el caso de los Lineamientos de Libre Competencia, OSIPTEL ya había señalado que la aplicación de las normas de competencia solo se produciría "en el supuesto que una determinada práctica o conducta no esté tipificada o cubierta de manera expresa por la normatividad específica del sector." ${ }^{\prime 32}$
Permitir ciertos escenarios en los que sea posible aplicar tanto las normas de competencia como la regulación sectorial genera impredecibilidad. Asimismo, dicha situación le otorgaría un gran poder a las agencias de competencia. Éstas no solo tendrían la potestad de resguardar el proceso competitivo mediante la aplicación de normas de competencia, pues también tendrían la posibilidad de evaluar la naturaleza de las disposiciones emitidas por los reguladores y sancionar conductas que deberían ser de su competencia.

Al respecto, en la línea de lo establecido por la doctrina de la inmunidad implícita estadounidense, Patrón sostiene lo siguiente:

"Lo realmente peligroso del criterio postulado por la Sala en la Resolución comentada [caso Alfagas] es el rol que indirectamente se termina atribuyendo el INDECOPI. Lo que en buena cuenta termina haciendo la Sala es otorgarle a los órganos funcionales del INDECOPI el poder para sancionar conductas sujetas a la supervisión y fiscalización de entidades regulatorias sectoriales especializadas, incluso contradiciendo los mandatos de estas últimas.

(...)

De este modo, INDECOPI se coloca como una especie de supraorganismo regulador de todos los sectores de la actividad económica que no se caractericen por ser monopolio natural." ${ }^{\prime 3}$

Como se observa, el autor sugiere separarse del criterio establecido en el caso Alfagas en la medida que, como sucede en la Unión Europea, la solución propuesta le otorgaría a las agencias de competencia el poder para fiscalizar conductas que se encuentran dentro del ámbito de competencia de entidades regulatorias.

31. Resolución N 0004-2008/TDC-INDECOPI del 3 de enero de 2008.

32. Numeral 4 de los Lineamientos de Libre Competencia.

33. Carlos Patrón, Op. Cit., 408. 
Por tales motivos, resulta pertinente excluir la aplicación de las normas de competencia en los casos en los que una conducta se encuentre tipificada por la regulación sectorial, sin importar la naturaleza de la normativa que establece la tipificación.

\section{IV. ¿SE CONCEPTUALIZÓ ADECUADAMENTE LOS ALCANCES DEL PRINCIPIO DE SUPLE- TORIEDAD EN EL CASO SPEEDY NAKED?}

De acuerdo con el argumento presentado por Telefónica en este caso, la comercialización de los servicios de telefonía fija y acceso a internet vía ADSL de manera conjunta corresponde a la situación descrita en el numeral (a) correspondiente a la sección previa. Es decir, la conducta imputada habría sido aprobada por la regulación sectorial. En ese sentido, el concepto propuesto por el Tribunal se centra en dicho supuesto, y establece lo siguiente:

"En consecuencia, en el presente caso, a fin de determinar si corresponde aplicar la normativa de libre competencia a la práctica de venta atada materia de investigación, debe determinarse lo siguiente:

a) si la práctica ha sido aprobada o impuesta por la regulación de una forma que resulte incompatible la aplicación de las normas de libre competencia, en cuyo caso se aplicará la regulación y no las normas de libre competencia; 0

b) si la regulación sectorial sólo ha constata- do el hecho de que la práctica es realizada en el mercado o no ha emitido opinión, al tratarse de una iniciativa empresarial privada, en cuyo caso aplicarán las normas de libre competencia." ${ }^{\prime \prime 4}$

Con relación a las conductas en las que la regulación sectorial aprueba o impone una conducta a los agentes del mercado, el Tribunal precisó que resulta necesario analizar el alcance de la aprobación efectuada, en tanto esta puede ser:

"(i) sólo "formal", con poca consideración de las consecuencias sobre la competencia, o (ii) si ha implicado un proceso de revisión exhaustivo". Teniendo en consideración dichas posibilidades, el Tribunal concluye que "(e)n el supuesto (i) se debe aplicar la normativa de libre competencia, en el supuesto (ii) debe ser descartada." ${ }^{135}$

Por otro lado, con relación a las conductas que la regulación sectorial solo ha "constatado", el Tribunal determina que deberán encontrarse sujetas a las normas de competencia puesto que "no resulta posible entender que ha operado una convalidación tácita que ha inmunizado dicha conducta de la aplicación de las normas de libre competencia. Por el contrario, en estos casos debe entenderse que, se trata de una materia sujeta al acuerdo de voluntades entre las partes y a las reglas del mercado (...)." ${ }^{\prime \prime 3}$

En ese sentido, la categorización propuesta por el Tribunal se puede resumir de la siguiente manera:
Supuestos

\begin{tabular}{|c|c|c|}
\hline \multirow{2}{*}{$\begin{array}{l}\text { Práctica aprobada o impuesta } \\
\text { por la regulación sectorial }\end{array}$} & $\begin{array}{l}\text { Aprobación "formal" con poca } \\
\text { consideración sobre la competencia }\end{array}$ & Aplican \\
\hline & $\begin{array}{l}\text { Aprobación que ha implicado } \\
\text { un proceso de revisión exhaustivo }\end{array}$ & No aplican \\
\hline \multicolumn{2}{|c|}{ Práctica constatada por la regulación sectorial } & Aplican \\
\hline
\end{tabular}

34. Resolución Final, 47.

35. Ibíd.

36. Ibíd., 46.

\section{Normas de competencia}

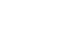


Como se observa, las categorías propuestas por el Tribunal no se desprenden de lo establecido en las normas o lineamientos que refieren al principio de supletoriedad, ni se condicen con la jurisprudencia en la materia. Por el contrario, el Tribunal ha optado por introducir una nueva categoría bajo la cual la regulación sectorial "constata" la realización de una conducta.

Sobre este primer punto, es importante notar que no resultaría posible establecer que la regulación sectorial pueda limitarse a "constatar" conductas en el mercado en los términos planteados por el Tribunal. Si se observa el criterio establecido en los Lineamientos, bajo el cual las conductas sujetas a aprobación o tipificación regulatoria deberán encontrarse señaladas expresamente, en términos logísticos, no podría presentarse una situación de mera "constatación" como sugiere el Tribunal.

Con relación a las conductas que son aprobadas o impuestas por la regulación, se puede apreciar que el Tribunal, sin referirse expresamente a ello, ha propuesto soluciones que parecen responder a la naturaleza de los bienes protegidos por las aprobaciones regulatorias que pueden presentarse, como sucede en el caso Alfagas. Al referirse a aprobaciones que han implicado una revisión exhaustiva sobre los efectos de la conducta en la competencia, parece referirse a aprobaciones regulatorias emitidas en el marco de normativa económica o para la competencia.

Por otro lado, al referirse a aprobaciones que han tenido "poca consideración" sobre su impacto en la competencia, parece referirse a aprobaciones o imposiciones emitidas en el marco de normas de naturaleza social, es decir, que buscan proteger bienes jurídicos distintos a las normas de competencia. Por tal motivo, opta por excluir la aplicación de normas de competencia en los supuestos en los que las normas que regulan la aprobación no tienen consideración por el proceso competitivo.

No obstante, como se señaló en la sección previa, cuando se trata de conductas aprobadas por la regulación, no se deberá aplicar las normas de competencia. Ello, con independencia del nivel de consideración en términos de competencia otorgado por el regulador que emitió la aprobación.

En efecto, los agentes económicos que actúan amparados bajo una autorización regulatoria despliegan sus actividades con la confianza de que, al haber recibido una aprobación, la conducta no podrá ser sancionada por normas de naturaleza distinta. Todo ello bajo el entendido de que la aprobación emitida responde a un diseño normativo que le brinda mayor relevancia a un bien jurídico distinto a la competencia. En ese sentido, no resulta coherente plantear la posibilidad de sancionar dicho tipo de conductas.

Por todo ello, la conceptualización efectuada por el Tribunal no resulta congruente con la jurisprudencia y la doctrina con relación al principio de supletoriedad. Es importante notar que el hecho de emitir una conceptualización errada del principio no es un aspecto menor. En efecto, en este caso, dicha situación supuso la aplicación de normas de competencia a una conducta que había sido aprobada de manera previa por la regulación aplicable. Como señalamos en la siguiente sección, tal aplicación habilitó a los órganos resolutivos de controversias de OSIPTEL a "corregir" la aprobación emitida por la Gerencia General.

\section{EFECTO DE LA APLICACIÓN DEL PRINCI- PIO DE SUPLETORIEDAD SEGÚN EL CRI- TERIO DEL TRIBUNAL}

Como se había mencionado previamente, a lo largo del procedimiento, Telefónica sostuvo que la venta conjunta de los servicios había sido autorizada a través de la emisión de una aprobación a los Contratos de Abonados que contenían cláusulas expresas que requerían de una línea fija de Telefónica como condición para adquirir el servicio Speedy. A continuación, se analiza a mayor detalle cómo es que dicha aprobación habría operado en el mercado, y por qué es que ésta debió haber excluido la aplicación de las normas de competencia. 
Las disposiciones relativas a la aprobación de los contratos de abonados que se encontraban vigentes al momento en que se produjo la aprobación regulatoria se encontraban en las Condiciones de Uso de los Servicios Públicos de Telecomunicaciones aprobadas por Resolución de Consejo Directivo No 116-2003-CD-OSIPTEL —en adelante, las "Condiciones de Uso"- . De acuerdo con lo establecido en tal norma, su finalidad era regular las relaciones entre los abonados y usuarios, frente a las empresas operadoras:

\section{"Artículo 1.- Objeto de la Norma}

La presente norma establece las obligaciones y derechos de las empresas operadoras, abonados y usuarios de servicios públicos de telecomunicaciones, y constituye el marco normativo general dentro del cual se desenvolverán las relaciones entre ellos."

En ese sentido, tomando en consideración la asimetría informativa, las Condiciones de Uso establecen disposiciones para subsanar dichas fallas y, con ello, garantizar el bienestar de los abonados y usuarios. Es por tal motivo que el artículo 11 de la norma establece requisitos para la correcta elaboración de los Contratos de Abonados, así como la información mínima que debe incluirse en ellos. Del mismo modo, la norma dispone lo obligación de remitir a OSIPTEL una copia del contrato para su aprobación:

"Artículo 11.- Cláusulas generales y adicionales de contratación

El contrato de abonado estará compuesto por las Cláusulas Generales de Contratación aprobadas por OSIPTEL y por aquellas cláusulas adicionales en las que el abonado consigne su opción respecto de cualesquiera de las alternativas de adquisición, arrendamiento $u$ otra modalidad de utilización de equipos, su mantenimiento u otras condiciones inherentes al servicio.

(...)

En los contratos de abonado deberá constar expresamente, como mínimo, la información a que se refieren los numerales (i), (iv), (v), (vi), (viii) y (ix) del artículo 6.

La empresa operadora deberá remitir a
OSIPTEL una copia del modelo de contrato de abonado, con anterioridad a la fecha de inicio de la comercialización del servicio, así como cualquier modificación al contenido del mismo."

La justificación para la inclusión de tales disposiciones fue incluida en la Exposición de Motivos de la norma, en la cual se establece expresamente que el propósito de que los contratos sean remitidos a OSIPTEL es que dicha entidad verifique el cumplimiento de las disposiciones contenidas en las Condiciones de Uso. Al respecto, se había establecido lo siguiente:

"También, se establece que las empresas operadoras están obligadas a remitir a OSIPTEL una copia de los modelos de contrato antes que el servicio sea comercializado, con la finalidad que éstos sean revisados y en caso éstos no se hubieran adecuado a las Condiciones de Uso o estuvieran contraviniendo alguna disposición de las mismas, realizar las observaciones respectivas a dichos modelos de contratos."

Tomando ello en consideración, es claro que la revisión que debía efectuarse por OSIPTEL debía ser comprensiva con relación a las disposiciones contenidas en las Condiciones de Uso. Dicho análisis no debía pasar por alto la posibilidad de determinar la validez de efectuar una venta conjunta de los servicios de telefonía fija y acceso a internet vía ADSL. Ello en la medida de que al momento en el que se produjeron las aprobaciones, las Condiciones de Uso establecían una prohibición expresa a la existencia de ventas atadas. Dicha prohibición se fijó en los siguientes términos:

"Artículo 17.- Prohibición de ventas atadas Toda persona tiene derecho a contratar por separado cualquiera de los servicios que ofrece la empresa operadora, quedando prohibido que la contratación se condicione a la adquisición, arrendamiento o cualquier otra forma de utilización, de los materiales y/o equipos de propiedad de la empresa operadora y/o a la contratación de otros servicios públicos de telecomunicaciones; sin perjuicio

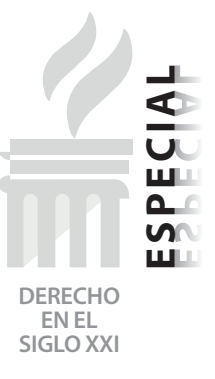

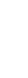


que la empresa operadora pueda ofrecer planes tarifarios, ofertas o promociones, en los cuales se incluya dichos componentes adicionales."

Como es el caso del artículo 11, la justificación para la inclusión de dicha prohibición fue expresada en la Exposición de Motivos de la norma, en la cual se establece lo siguiente:

"La presente norma establece que al momento de la contratación, los abonados pueden optar por la adquisición a la empresa operadora o a un tercero ajeno a ella, de los equipos terminales y de la instalación de los mismos. Siendo el único requisito que los referidos equipos se encuentren debidamente homologados por el Ministerio de Transportes y Comunicaciones.

(...)

Igualmente, esta norma establece la prohibición de condicionar la contratación del servicio a la adquisición, arrendamiento o cualquier otra forma de utilización de los materiales y/o equipos de titularidad de las empresas operadoras y/o a la contratación de otros los servicios públicos de telecomunicaciones. Con ello, no se prohíbe a las empresas operadoras a ofrecer planes tarifarios, ofertas o promociones en los que se incluyan los referidos componentes. Lo importante es que los solicitantes de los servicios tengan la posibilidad de elegir qué propuesta responde mejor a sus necesidades."

Sin perjuicio de lo señalado en la normativa, el Tribunal indicó que no resultaba razonable que Telefónica entendiera, de buena fe, que la conformidad dada por la Gerencia General en virtud de lo establecido en el artículo 11 de Condiciones de Uso constituía también la opinión de las instancias de solución de controversias en materia de libre competencia. Mucho menos, que ello significara que éstas se inhibirían de iniciar cualquier procedimiento destinado a investigar los efectos de esta oferta comercial. ${ }^{37}$
No obstante, una aplicación adecuada y rigurosa del principio de supletoriedad en los términos definidos en el presente trabajo nos permite llegar a una conclusión diferente. Al respecto, el elemento clave en el análisis del Tribunal debió ser la evaluación de la existencia de una aprobación que pudiera ser considerada como "expresa", de acuerdo con lo señalado en la sección previa y en concordancia con los Lineamientos de Libre Competencia.

Es relevante reiterar que, en los casos de aprobación de conductas, no resulta relevante tomar en consideración la naturaleza del bien jurídico protegido a través de la normativa que regula o dispone la aprobación. Ello en la medida que, al aprobar una conducta determinada, debemos entender que la regulación sectorial ha optado por primar un bien jurídico distinto a la competencia en un caso concreto.

Al respecto, se puede apreciar que sí existió una aprobación expresa a la conducta objeto de análisis mediante el presente procedimiento en la medida que: (a) los Contratos de Abonados referían a la necesidad de contar con una línea contratada con Telefónica como un requisito para la adquisición del servicio de acceso a internet vía ADSL; (b) la exposición de motivos del artículo 11 de las Condiciones de Uso dispone que el objetivo de la verificación de OSIPTEL es constatar la legalidad de los Contratos de Abonados; y, (c) todos los Contratos de Abonados fueron aprobados a pesar de la existencia de una prohibición expresa en las Condiciones de Uso para las ventas atadas.

Tomando todo ello en consideración, en el presente caso sí concurrió una aprobación que excluía las normas de competencia a través de las cartas No 082-GG.GUS/2009 y N 489-GG. GUS/2009, por las cuales OSIPTEL manifestó su conformidad con los Contratos de Abonados. El hecho de que se prohibiera la conducta a pesar de la existencia de dichas aprobaciones

37. Resolución Final, 56. 
determinó una indebida atribución de facultades para modificar la decisión de la Gerencia General.

Del mismo modo, incluso en el supuesto que de que los Contratos de Abonados no comprendieran una aprobación expresa a la comercialización conjunta de los servicios, de todos modos hubiese correspondido que en aplicación del principio de supletoriedad se excluyera la aplicación de las normas de competencia. En efecto, teniendo en consideración que en este caso el artículo 17 de las Condiciones de Uso establece la tipificación de la conducta tipificada por las normas de competencia, lo pertinente hubiese sido declarar la improcedencia de la denuncia, e iniciar el procedimiento de índole regulatorio para cuestionar la práctica.

El Tribunal determinó que la prohibición regulatoria a las ventas atadas contenida en el artículo 17 de las Condiciones de Uso no excluía la aplicación de las normas de competencia. Para ello, realizó una diferenciación del bien jurídico tutelado mediante dicha disposición, el derecho de cada consumidor a decidir qué productos adquiere y qué servicios contrata en el mercado, frente al bien jurídico protegido en el caso de las normas de competencia, el proceso competitivo.

Asimismo, tomando en consideración los elementos necesarios para configurar el tipo en cada infracción mencionó:

"(...) para que se configure un supuesto de venta atada tipificado en las normas de libre competencia, no solo es necesario que se verifique la existencia de una venta atada, en tanto coerción para el comprador, sino que es necesario que se acredite que dicha conducta ha generado efectos exclusorios en el mer- cado que superen las eficiencias que podría haber producido. ${ }^{38}$

(...) no resulta razonable interpretar que la conformidad del OSIPTEL haya implicado que la atadura implementada por TELEFÓNICA (...) no podía ser objeto de investigación y sanción bajo las normas de libre competencia (...) cuyo bien jurídico protegido, requisitos configurantes del tipo administrativo y metodología de evaluación son diferentes a los empleados por las normas de protección al consumidor." ${ }^{139}$

Sin embargo, lo que revela el análisis efectuado por el Tribunal con relación a los elementos del tipo de cada infracción es que la conducta prohibida por la regulación resultaba más comprensiva en su ámbito de prohibición frente a lo establecido en la normativa de competencia. En ese escenario, resulta aún más razonable excluir la aplicación de las normas de competencia. La existencia de una regulación sectorial más comprensiva eliminó cualquier "espacio vacío" en el que se podría requerir la fiscalización de la agencia de competencia.

Por todo lo señalado, las aprobaciones a los Contratos de Abonados sí constituyeron aprobaciones regulatorias que debieron excluir la aplicación de las normas de libre competencia. Tales Contratos de Abonados refieren de manera expresa al condicionamiento de la prestación del servicio de acceso a internet vía ADSL a la tenencia previa de una línea de telefonía fija de Telefónica, y fueron analizados en contraste con la prohibición a las ventas atadas contenida en el artículo 17 de las Condiciones de Uso. Del mismo modo, aún si no hubiese existido una aprobación, hubiese correspondido la exclusión de las normas de competencia debido a que la conducta investigada se encontraba tipificada en el artículo

38. Ibíd., 54-55.

39. Ibíd., 56. 
17 de las Condiciones de Uso. Por ello, el cuestionamiento a la conducta debió formularse a través de la vía regulatoria ${ }^{40}$.

Finalmente, es importante notar que los efectos de la decisión del Tribunal en el presente caso no se limitaron a afectar la situación de Telefónica. En efecto, el criterio del Tribunal para definir los alcances del principio de supletoriedad ha sido plasmado en los nuevos Lineamientos Generales para la Aplicación de las Normas de Represión de Conductas Anticompetitivas en el Ámbito de las Telecomunicaciones, aprobados por Resolución No 077-2016-CD/OSIPTEL. Con ello, se ha incluido la posibilidad de injerir en las decisiones de otros reguladores a través de la aplicación de dicho criterio.

\section{A MODO DE CONCLUSIÓN}

La correcta conceptualización y aplicación del principio de supletoriedad es de gran importancia para el ordenamiento jurídico puesto que es un mecanismo que le brinda coherencia, proporcionando respuestas para conflictos normativos entre las normas de competencia y la regulación. Sin embargo, la jurisprudencia no ha sido consistente en las condiciones que deben presentarse a fin de que concurra la aplicación del principio.

Sin perjuicio de ello, atendiendo al principio de especialidad de las normas, la coherencia del ordenamiento $y$, principalmente, a las expectativas de los agentes que actúan bajo el amparo de mandatos regulatorios, el principio de supletoriedad debe implicar la exclusión de las normas de competencia, siempre que exista una norma específica que tipifique o apruebe la conducta objeto de análisis de manera expresa. Ello, con independencia de los bienes protegidos o de la naturaleza de la regulación que refiere a la conducta cuestionada.
Si bien la postura mencionada es ampliamente aceptada para el caso de las conductas que son aprobadas por la regulación, la Resolución Final se aparta injustificadamente de este criterio. De acuerdo con lo establecido por el Tribunal, es posible sancionar a través de un procedimiento de libre competencia una conducta que ha sido previamente aprobada por la regulación, cuando tal aprobación haya sido emitida sin observar su impacto en la competencia. Como se ha mencionado previamente, dicho pronunciamiento genera incongruencias en el sistema jurídico y resta predictibilidad a las actuaciones de los administrados.

En el caso específico, la aplicación del principio de supletoriedad en los términos del Tribunal determinó la aplicación de normas de competencia a una conducta que se encontraba autorizada previamente por la Gerencia General de OSIPTEL. Siendo ello así, al prohibir una conducta previamente aprobada, tanto el Cuerpo Colegiado como el Tribunal se adjudicaron la facultad de modificar las decisiones de la Gerencia General. En otras palabras, se utilizó un procedimiento de libre competencia para "obviar" la autorización otorgada a una práctica.

Es necesario repensar la manera en la que se delimita los alcances del principio de supletoriedad a fin de brindar mayor unidad al sistema jurídico; y, sobre todo, evitar situaciones en las que se le pueda otorgar facultades indebidas a las agencias de competencia. Si bien este trabajo se ha centrado en el sector telecomunicaciones, esta labor deberá ser aplicada de manera uniforme en todos los mercados cuya regulación pueda entrar en conflicto con las normas de competencia. Los futuros pronunciamientos en la materia deberán establecer criterios dirigidos a brindar mayor seguridad jurídica a los agentes económicos.

40. Pocos meses después de la emisión de la Resolución Final, la regulación sectorial relativa a los esquemas habilitados a las operadoras para la comercialización conjunta de servicios fue modificada. En efecto, mediante una incorporación en las disposiciones en materia tarifaria, el OSIPTEL estableció reglas complementarias dirigidas a reiterar la prohibición a las ventas atadas ya consignadas en las Condiciones de Uso, así como incorporar reglas en materia tarifaria para evitar el establecimiento de empaquetaduras. En términos prácticos, por la vía regulatoria, dicha modificación otorgó efectos generales a la medida correctiva impuesta a través del procedimiento de libre competencia. 